\title{
Multiple origins of the spinocerebellar ataxia 7 (SCA7) mutation revealed by linkage disequilibrium studies with closely flanking markers, including an intragenic polymorphism $\left(G^{3145} T G / A^{3145} T G\right)$
}

G Stevanin ${ }^{1}$, G David ${ }^{1}$, A Dürr ${ }^{1,2,3}$, P Giunti $^{4}$, A Benomar ${ }^{5}$, M Abada-Bendib ${ }^{6}$, M S Lee ${ }^{7}$, Y Agid $^{1,2}$ and A Brice ${ }^{1,2,3}$

${ }^{1}$ INSERM U289, Hôpital de la Salpêtrière

${ }^{2}$ Fédération de Neurologie, Hôpital de la Salpêtrière

${ }^{3}$ Consultation de Génétique Médicale, Hôpital de la Salpêtrière, Paris, France

${ }^{4}$ Institute of Neurology, Queen's Square, London, UK

${ }^{5}$ Service de Neurologie (Prof. T Chkili), Hôpital des Spécialités, Rabat, Morocco

${ }^{6}$ Service de Neurologie, Hôpital de Ben-Aknoum, CHU Alger-Ouest, Algiers, Algeria

${ }^{7}$ Department of Neurology, Yongdong Severance Hospital, Seoul, Korea

Spinocerebellar ataxia 7 (SCA7) is a neurodegenerative disease characterised by the association of cerebellar ataxia and, in most patients, progressive macular degeneration leading to loss of autonomy and blindness. The patients die after 5-30 years of evolution. The cause of the disease has been identified as a (CAG) $)_{n}$ repeat expansion in the coding sequence of the SCA7 gene on chromosome 3p. De novo mutations occur on intermediate-sized alleles carrying from 28 to 35 CAG repeats. Neomutations explain the persistence of the disease in spite of the great instability of the repeat sequence which results in the appearance of juvenile onset patients and the extinction of the disease within families. This rare disorder has been reported in a wide variety of countries and ethnic groups. In a large number of SCA7 families $(n=41)$ of different origins, we have determined the haplotypes segregating with the mutation of several microsatellite markers close to the $S C A 7$ gene and of a new intragenic polymorphism $\left(G^{3145} T G / A^{3145} T G\right)$. Four different haplotypes were found for centromeric markers $\left(G^{3145} T G / A^{3145} T G-D 3 S 1287-D 3 S 3635\right)$ in the majority of the kindreds from four different geographic regions: $A-2-4$ in Korea; $A-3-6$ in North Africa, $B-3-6$ in continental Europe and A-4-6 in the UK and USA. The haplotypes in the Jamaican, Filipino, Brazilian and German families were different, suggesting that independent regional founders are at the origin of the $S C A 7$ mutation in each population. Two different haplotypes were observed, however, in two families from the same rural area in central Italy in which de novo SCA7 mutations on intermediate alleles have been observed, suggesting the existence of different pools of at-risk chromosomes in this population.

Correspondence: Professor Alexis Brice, INSERM U289, Hôpital de la Salpêtrière, 47 Bd de l'Hôpital, 75651 Paris Cedex 13, France. Tel: 0033142162182 ; Fax: 0033144243658 ; E-mail: brice@ccr.jussieu.fr

Received 21 May 1999; revised 29 July 1999; accepted 4 August 1999 
Keywords: chromosome 3; spinocerebellar ataxia 7; autosomal dominant cerebellar ataxia type II; macular dystrophy; linkage disequilibrium; single nucleotide polymorphism; microsatellite; founder effect

\section{Introduction}

Spinocerebellar ataxia 7 (SCA7) is a rare form of autosomal dominant cerebellar ataxia (ADCA). ${ }^{1}$ Patients suffer from unremitting cerebellar ataxia associated with progressive macular dystrophy. ${ }^{2-5}$ The molecular defect involved in this disease has been identified as the abnormal expansion of a (CAG) repeat in the coding region of the $S C A 7$ gene on chromosome $3 .^{6-8}$ The same type of mutation has been implicated in other neurogenerative disorders such as Huntington's disease, spinobulbar muscular atrophy, dentatorubro-pallidoluysian atrophy, as well as in other genetic subtypes of ADCA: SCA1, SCA2, SCA3/MJD (Machado-Joseph disease) and SCA6. ${ }^{9}$

$S C A 7$ is a minor mutation that accounts for 5 to $12 \%$ of ADCA, ${ }^{8,10,11}$ but is found in a wide variety of countries and in patients of many ethnic origins. ${ }^{7,12-18}$ We describe here a new intragenic polymorphism of the $S C A 7$ gene $\left(G^{3145} T G / A^{3145} T G\right)$. It has been analysed in combination with the haplotypes of closely linked microsatellite markers in a large series of families with SCA7.

\section{Materials and Methods}

\section{Patients and Families}

Non-SCA1,2,3 and six patients with autosomal dominant cerebellar ataxia and sporadic cases were screened for the SCA7 mutation. Thirty-nine unrelated families and two isolated patients ${ }^{19,20}$ were identified representing about $6 \%$ of ADCA and less than $1 \%$ of isolated ataxias. ${ }^{11}$ Twenty of these cases, five of which were recruited in the USA, were European Caucasians (six were from France, 10 from the UK, two from Italy and one each from Belgium and Germany). Thirteen kindreds were non-black North Africans (seven from Morocco, five from Algeria and one from Tunisia). Four families were Asian (three from Korea and one from the Philippines). A kindred, of Jewish ancestry, originated from the Arabian peninsula. One each were from Jamaica, Brazil and South Africa but their ethnic background could not be precisely determined. The clinical and genetic profiles of 38 of these kindreds, including partial haplotypes in 16, have been previously reported. ${ }^{12,15,16,19-21}$

\section{Intragenic Polymorphism}

The SCA7 cDNAs clones D1 and $A 10,{ }^{6}$ obtained from different cDNA libraries generated from two lymphoblastoid cell lines of ataxic patients, ${ }^{22}$ diverged at position 3145 $\left(G^{3145} T G / A^{3145} T G\right)$, resulting in a Val to Met substitution at amino acid 862 of the encoded protein (GDB:9866030). The alleles differ by the absence or the presence of a restriction site for NlaIII. Patients were screened for this polymorphism as follows. The region containing the polymorphism was first amplified by PCR using primers sca7-3060-F (AGGAAAGAAAAGAAAGTG) and sca7-3208-R (TGGCTGAT$G A A G G A G G G A), 25 \mathrm{pmol}$ each, in a final volume of $25 \mu \mathrm{l}$ containing $200 \mathrm{ng}$ of genomic DNA, $240 \mu \mathrm{M}$ of each dNTP and $1 \times$ PCR buffer. One unit of Taq DNA polymerase (Perkin Elmer Cetus, Foster City, CA, USA) was added during the first denaturation step $\left(10 \mathrm{~min}\right.$ at $\left.94^{\circ} \mathrm{C}\right)$, followed by 35 cycles of denaturation $\left(94^{\circ} \mathrm{C}, 15 \mathrm{~s}\right)$, annealing $\left(55^{\circ} \mathrm{C}, 15 \mathrm{~s}\right)$ and extension $\left(72^{\circ} \mathrm{C}, 15 \mathrm{~s}\right.$ extended to $2 \mathrm{~min}$ for the final extension step) in a Gene Amp PCR system (Perkin Elmer Cetus, Foster City, CA, USA). PCR products (166 bp) were then digested for 3 hours at $37^{\circ} \mathrm{C}$ with 6 units of NlaIII in a final volume of $30 \mu \mathrm{l}$ in the supplier's buffer (New England Biolabs Inc, Beverly, MA, USA). The digestion products were separated by electrophoresis on a $1 \%$ agarose $/ 2 \%$ metaphore gel (FMC bioproducts, Rockland, ME, USA) stained with $0.5 \mu \mathrm{g} / \mathrm{ml}$ ethidium bromide and visualised under UV light. A $68 \mathrm{bp}$ fragment was generated in all subjects because of a NlaIII site at position 3158. The second NlaIII site at position 3144 resulted in the cleavage of the remaining $98 \mathrm{bp}$ sequence into two fragments of 84 and $14 \mathrm{bp}$, defined as allele $A\left(G^{3145} T G\right)$. Allele $B\left(A^{3145} T G\right)$ did not contain this restriction site. Allele frequencies were determined in 69 unrelated healthy individuals, 48 of whom were northwestern Caucasian Europeans and 21 were non-black North Africans. The position of this polymorphism relative to other markers and to the $(\mathrm{CAG})_{\mathrm{n}}$ repeat sequence was determined by PCR typing of 19 overlapping YACs from the SCA7 region. ${ }^{12}$

\section{Microsatellite Markers and Genotyping}

Genotypes were determined for six microsatellite markers spanning about $8 \mathrm{cM}$ on chromosome $3 \mathrm{p}^{12,21}$ S3566 (2 cM)-S3698 (1 cM)-S1600 (2.5 cM)-S1287 (2 cM)-S3635 $(<1 \mathrm{cM})-S 3644$. SCA7 is distal to D3S1287 and proximal to D3S1600. ${ }^{7}$ Each marker was amplified by PCR and genotypes were determined using allele numbering as described. ${ }^{12,21}$ Allele frequencies were determined from at least 63 control individuals mostly of European or North African origin. $G^{3145} T G / A^{3145} T G-D 3 S 1287-D 3 S 3635$ haplotype frequencies were calculated in 32 healthy married-in members of the SCA7 families, including 10 of North African origin and 18 of continental European descent.

\section{Linkage Disequilibrium Analysis}

Allele distribution on normal and carrier chromosomes was compared at each locus by the two-tailed Fisher's exact test, and the Yates corrected $\chi^{2}$ test when necessary. Only the ancestral disease-linked allele or haplotype of each family was used if it could be determined. The frequency of each allele was compared with a pool of all the others treated as a second allele. The presence of linkage disequilibrium was tested with the formula $D_{\text {st }}=\sqrt{ }\left(\chi^{2} / N\right)$ using the uncorrected 
$\chi^{2}$ value. ${ }^{23}$ Comparison of means was performed using the non-parametric Mann Whitney-U test.

\section{Results}

\section{Analysis of the $\mathrm{G}^{3145} \mathrm{TG} / \mathrm{A}^{3145} \mathrm{TG}$ Intragenic Polymorphism}

The frequencies of alleles $A$ and $B$ of the polymorphism, determined by PCR and NlaIII digestion on 69 healthy unrelated individuals, were 31 and 69\%, respectively (Table 1 ). The observed heterozygosity rate was $35 \%$ in the whole population. This was lower, although not significantly, than the expected $43 \%$ heterozygosity given the allele frequencies. The relative proportions of each allele in European and North African populations differed significantly $(P<0.05$, Table 1).

The allele of the intragenic polymorphism segregating with the SCA7 mutation was determined in 34 of
41 families and the presence of linkage disequilibrium (LD) between the polymorphism and the CAG repeat expansion was examined. Allele $A$ segregated with the disease in 27/34 of the SCA7 kindreds and was significantly more frequent in patients than in controls, in the whole sample $(P<0.001)$ and in the North African and European populations $(P<0.05$, Table 2$)$. Allele $A$ was in complete linkage disequilibrium with the mutations in North Africa and also segregated with the mutation in the four Asian kindreds (Table 3). In

Table 1 Distribution of the $G^{3145} T G / A^{3145} T G$ alleles according to geographical origin (number of chromosomes)

\begin{tabular}{llll}
\hline & \multicolumn{3}{c}{ Origins } \\
\cline { 2 - 4 } Allele & All & Europe & North Africa \\
\hline$A$ & 43 & 24 & 19 \\
$B$ & 95 & 72 & 23 \\
\hline
\end{tabular}

Table 2 Linkage disequilibrium of the $S C A 7$ mutation with four chromosome $3 p$ markers (A) and the haplotype $G^{3145} T G / A^{3145} T G-D 3 S 1287-D 3 S 3635$ (B)

\begin{tabular}{|c|c|c|c|c|c|c|c|}
\hline Locus & Allele & Origin & $n_{\text {patients }} / N$ & $n_{\text {controls }} / N$ & $\chi^{2}$ & Significance $(P=)$ & $D_{\text {st }}$ \\
\hline \multirow[t]{4}{*}{ D3S1600 } & 5 & All & $9 / 31$ & $20 / 132$ & 3.31 & NS (0.064) & 0.14 \\
\hline & 13 & All & $9 / 31$ & $32 / 132$ & 0.31 & NS (0.366) & 0.04 \\
\hline & 13 & Europe* & $7 / 15$ & $13 / 60$ & 2.66 & NS (0.098) & 0.23 \\
\hline & 6 & North Africa & $4 / 12$ & $3 / 42$ & 3.59 & NS (0.058) & 0.32 \\
\hline \multirow[t]{3}{*}{$G^{3145} T G / A^{3145} T G$} & A & All & $27 / 34$ & $43 / 138$ & 26.32 & $<0.001$ & 0.39 \\
\hline & A & Europe* & $8 / 14$ & $24 / 96$ & 4.66 & 0.024 & 0.24 \\
\hline & A & North Africa & $11 / 11$ & $19 / 42$ & 8.53 & 0.001 & 0.45 \\
\hline \multirow[t]{3}{*}{ D3S1287 } & 3 & All & $18 / 31$ & $54 / 128$ & 2.54 & NS (0.159) & 0.13 \\
\hline & 3 & Europe* & $8 / 14$ & $29 / 64$ & 0.65 & NS $(0.557)$ & 0.10 \\
\hline & 3 & North Africa & $10 / 11$ & $33 / 42$ & 0.25 & NS (0.618) & 0.13 \\
\hline \multirow[t]{3}{*}{ D3S3635 } & 6 & All & $25 / 35$ & $67 / 130$ & 4.42 & 0.037 & 0.16 \\
\hline & 6 & Europe* & $14 / 17$ & $33 / 62$ & 4.70 & 0.049 & 0.24 \\
\hline & 6 & North Africa & $11 / 13$ & $18 / 38$ & 5.48 & 0.025 & 0.33 \\
\hline \multirow[t]{3}{*}{ D3S3644 } & 6 & All & $27 / 37$ & $66 / 126$ & 4.95 & 0.037 & 0.17 \\
\hline & 6 & Europe* & 9/18 & $30 / 62$ & 0.01 & NS (1.000) & 0.01 \\
\hline & 6 & North Africa & $11 / 13$ & $18 / 34$ & 3.99 & NS $(0.091)$ & 0.29 \\
\hline
\end{tabular}

B

\begin{tabular}{lllllll}
\hline Haplotype & Origin & $n_{\text {patiens }} / N$ & $n_{\text {controls }} / N$ & $\chi^{2}$ & Significance $(P=)$ & $D_{\text {st }}$ \\
\hline$A-3-6$ & All & $10 / 29$ & $4 / 64$ & 10.33 & 0.001 & 0.37 \\
$A-3-6$ & North Africa & $8 / 9$ & $3 / 20$ & 11.43 & $<0.001$ & 0.70 \\
$A-4-6$ & Anglo-Saxons & $3 / 4$ & $3 / 36$ & 7.86 & 0.005 & 0.56 \\
$B-3-6$ & Continental Europe & $4 / 8$ & $4 / 36$ & 4.30 & 0.038 & 0.39
\end{tabular}

$\mathrm{D}_{\mathrm{st}}$ : coefficient of disequilibrium $\mathrm{D}_{\mathrm{st}}=\left(\chi^{2} / N\right)$, where $N$ is the number of families in which the segregating allele could be determined, or the number of chromosomes used for the calculation.

*These results were not significant if the calculations were performed on continental Europeans only

NS: not significant 
European SCA7 kindreds, allele $A$ segregated with the disease in a German, an Italian, and a French family and all but one Anglo-Saxon North American and English kindreds (Table 3, $P<0.05$ ). Allele $B$ segregated with the disease in all but one of the French families, a French kindred of Belgian descent, an Italian and an English family, but was not observed on diseasebearing chromosomes in SCA7 families of other origins. Interestingly, the Italian families carried different alleles on the pathological chromosome, although they were from the same rural area of central Italy.

On normal chromosomes, allele $A$ was associated with a significantly $(P<0.001)$ larger number of CAG repeats $(11.0 \pm 1.4 \mathrm{CAG}$ repeats, from 7 to $13, n=39)$ than allele $B(10.0 \pm 0.4$ repeats, from 9 to $12, n=57)$, a feature that remained significant when European and North African populations were analysed separately. Alleles carrying $10 \mathrm{CAG}$ repeats represented $46 \%$ of those associated with polymorphism $A$ and $95 \%$ of those with polymorphism $B$. In European controls, all the alleles with polymorphism $B$ were associated with $10 \mathrm{CAG}$ repeats.

We found no relationships between the allele carried by the patients and their clinical presentation (age at onset, disease severity) or degree of intergenerational instability.

To establish the position of the $G^{3145} T G / A^{3145} T G$ polymorphism with respect to the $(\mathrm{CAG})_{\mathrm{n}}$ repeat sequence and flanking microsatellite markers, we screened the subset of YACs which constitutes a contig of the $S C A 7$ region by PCR. ${ }^{12}$ The $G^{3145} T G / A^{3145} T G$ polymorphism was present in 10 YACs. Marker D3S1287 could be amplified in all 10. Nine also carried the $(\mathrm{CAG})_{\mathrm{n}}$ repeat sequence. Only two were positive for marker $D 3 S 1600$. The order of markers is therefore tel $-D 3 S 1600-(\mathrm{CAG})_{\mathrm{n}}-G^{3145} T G / A^{3145} T G-D 3 S 1287$

Table 3 Partial haplotypes in 33 SCA7 families of different geographical origins

\begin{tabular}{|c|c|c|c|c|c|c|c|c|}
\hline Geographical origin & Country & D3S3566 & D3S3698 & D3S1600 & $G^{3145} T G / A^{3145} T G$ & D3S1287 & D3S3635 & D3S3644 \\
\hline Asia & Philippines & - & 6 & $5 / 13$ & A & 3 & 4 & 6 \\
\hline Asia & Korea & 4 & 6 & 5 & A & 2 & 4 & 6 \\
\hline Asia & Korea & 4 & 6 & 5 & A & 2 & 4 & 6 \\
\hline Asia & Korea & 4 & 7 & 13 & A & 2 & 4 & 6 \\
\hline North Africa & Algeria & 2 & 6 & 10 & A & 3 & 6 & 6 \\
\hline North Africa & Algeria & -1 & 8 & 13 & A & 3 & 6 & 6 \\
\hline North Africa & Morocco & 4 & 6 & 5 & $\mathrm{~A} / \mathrm{B}$ & 3 & 6 & 1 \\
\hline North Africa & Morocco & 5 & 6 & 5 & A & 3 & 6 & 6 \\
\hline North Africa & Morocco & 4 & 6 & 6 & A & 3 & 6 & 6 \\
\hline North Africa & Morocco & 4 & 6 & 6 & A & 3 & 6 & 6 \\
\hline North Africa & Morocco & 4 & 6 & 6 & A & 3 & 6 & 6 \\
\hline North Africa & Morocco & 4 & 6 & 12 & A & 3 & 6 & 6 \\
\hline North Africa & Morocco & 4 & 8 & 12 & A & 3 & 6 & 6 \\
\hline North Africa & Tunisia* & 4 & 8 & 6 & A & 4 & 6 & 6 \\
\hline Anglo-Saxons & USA & $4 / 6$ & 8 & $12 / 13$ & A & $2 / 4$ & 6 & 3 \\
\hline Anglo-Saxons & UK & 4 & 7 & 5 & A & 4 & 6 & 6 \\
\hline Anglo-Saxons & UK & 4 & 7 & 5 & A & 4 & 6 & 6 \\
\hline Anglo-Saxons & UK & 4 & 7 & 5 & A & 4 & $6 / 4$ & 6 \\
\hline Anglo-Saxons & UK & 4 & 6 & 13 & B & 4 & 6 & 2 \\
\hline Anglo-Saxons & UK & 4 & 6 & 13 & $\mathrm{~A} / \mathrm{B}$ & $3 / 4$ & 6 & $3 / 6$ \\
\hline Continental Europe & Italy* & 3 & 7 & 5 & B & 3 & 4 & 2 \\
\hline Continental Europe & Belgium & 4 & 4 & 13 & $\mathrm{~B}$ & 3 & 6 & 3 \\
\hline Continental Europe & France & -3 & 6 & 13 & $\mathrm{~B}$ & 3 & 6 & 3 \\
\hline Continental Europe & France & 4 & 7 & 13 & $\mathrm{~B}$ & 3 & 6 & 3 \\
\hline Continental Europe & France & 6 & 8 & 12 & B & 3 & 6 & 6 \\
\hline Continental Europe & France & 4 & 8 & 2 & A & 3 & 6 & 2 \\
\hline Continental Europe & Italy* & 4 & 8 & 10 & A & 3 & 6 & 3 \\
\hline Continental Europe & Germany & 4 & 6 & 2 & A & 5 & 10 & 6 \\
\hline Middle East & Arabian Peninsula & 5 & 8 & 5 & A & 4 & 5 & 6 \\
\hline- & South Africa & 2 & 6 & 13 & $\mathrm{~A} / \mathrm{B}$ & $2 / 10$ & 3 & 3 \\
\hline South America or Europe & Brazil & 4 & 6 & 11 & A & 2 & 6 & 6 \\
\hline
\end{tabular}

The SCA7 (CAG)n repeat is located between $D 3 S 1600$ and the $G^{3145} T G / A^{3145} T G$ polymorphism

${ }^{*}$ Families in which IA segregate or are at the origin of de novo expansion. ${ }^{18}$ 
- cen. Since fewer YACs carry D3S1600 rather than $D 3 S 1287$, the latter is probably closer to the SCA7 gene.

\section{Linkage Disequilibrium with Flanking Microsatellite Markers}

The SCA7 mutation was in significant linkage disequilibrium (LD) with two of the centromeric markers (Table 2). Allele 6 of D3S3635 was significantly more frequent in patients of all origins than in controls $(P<0.05)$. Although allele 6 was frequently found in European $(53 \%)$ and North African $(47 \%)$ controls, LD was significant in both groups $(P<0.05)$. Allele 6 of marker D3S3644 segregated more frequently in patients than in controls in the whole SCA7 population $(P<0.05)$, but its frequency did not differ significantly in each population independently.

We found no LD with the closely flanking loci. Allele 3 of marker D3S1287 segregated with the disease in 18/31 kindreds, but this allele was frequently detected in controls $(P>0.05)$. In North Africans, allele 3 of this marker segregated in 10/11 SCA7 families but it represented $79 \%$ of the normal chromosomes $(P>0.05)$. Finally, 7 different alleles of marker D3S1600 were found in SCA7 patients, but none were in significant LD $(P>0.05)$ with the $S C A 7$ mutation. This would be consistent with a distance between the marker and the expansion that is greater for D3S1600 than D3S1287, as suggested by the results of the YAC screening (see above).

\section{Disease-linked Haplotypes}

Complete or partial SCA7-carrier haplotypes could be reconstructed in 33/41 SCA7 kindreds (Table 3). Seventeen $D 3 S 1600-G^{3145} T G / A^{3145} T G-D 3 S 1287-D 3 S 3635$ haplotypes were observed in the SCA7 families. Families grouped according to their origin shared common haplotypes, particularly in the centromeric part of the $S C A 7$ region. Four major haplotypes of the centromeric markers $\left(G^{3145} T G / A^{3145} T G-D 3 S 1287-D 3 S 3635\right)$ accounted for $75 \%$ (21/28) of those associated with the disease and were found, with few exceptions, in four different geographic areas: $A-2-4$ in Korea, $A-3-6$ in North Africa, $B-3-6$ in continental Europe (France and Belgium) and $A-4-6$ in the UK and USA. The major haplotype $(A-3-6)$ was more frequently observed in the SCA7 kindreds than in controls $(P<0.01)$, particularly in those of North African descent $(P<0.001)$, as shown by the $\mathrm{D}_{\mathrm{st}}$ value of 0.70 (Table 2). In European kindreds, haplotype $B-3-6$ occurred more frequently in continental families $(P<0.05)$ whereas haplotype
A-4-6 was observed in kindreds from the UK and North America. Given the small number of families, the difference between normal and carriers chromosomes did not reach statistical significance for the other haplotypes.

The telomeric portion of the chromosome was less conserved among the families, even those of common origin, in this study as well as in four previously published Swedish kindreds. ${ }^{14}$ This could be explained by the position of the mutation that is probably closer to D3S1287 than to D3S1600. The haplotypes segregating in the German, Brazilian, Jamaican and Filipino pedigrees were different, suggesting independent ancestral mutations. In the Jamaican pedigree, the linked haplotype also diverged with respect to the number of $(\mathrm{CCG})_{\mathrm{n}}$ repeats present immediately $3^{\prime}$ to the $(\mathrm{CAG})_{\mathrm{n}}$ repeat. $^{6}$ Three repeats were present instead of the two usually found..$^{20}$ This variation was observed by direct sequencing with primers $4 U-1024$ and $4 U-716^{6}$ only in this kindred and on one control chromosome, but not on 10 normal, two intermediatesized or two pathological alleles (data not shown).

Interestingly, the linked haplotypes segregating in the two Italian families diverged both in their telomeric and centromeric regions surrounding the intragenic marker, except for allele 3 at D3S1287, which is frequent.

\section{Discussion}

We have described a new intragenic polymorphism $\left(G^{3145} T G / A^{3145} T G\right)$ that is in linkage disequilibrium with the $S C A 7$ mutation. Allele $A$ predominates in Anglo-Saxon (UK and USA), Asian and North African kindreds, allele $B$ in continental European families. We found that almost all alleles $B$, but only $50 \%$ of alleles $A$, are associated with chromosomes carrying $10 \mathrm{CAG}$ repeats in controls. This suggests that allele $B$ derived from an allele $A$ carrying 10 repeat units. Alternatively, allele $A$ might be associated with chromosomes that are more prone to instability and might explain why allele $B$ is rare on pathological chromosomes. However, although allele $A$ segregated in $79 \%$ of the SCA7 families, it was not correlated with a greater degree of instability in the SCA7 patients studied.

\section{Multiple Origins of the SCA7 Mutation?}

The intragenic polymorphism distinguishes haplotypes associated with the mutation in continental European from those in North African families. Four major 
haplotypes for markers $G^{3145} T G / A^{3145} T G$ - D3S1287 and D3S3635 were found in most families. This argues in favour of multiple origins for the $S C A 7$ mutation. The existence of different haplotypes in single families from Germany, the Arabian peninsula, South Africa, Brazil, Jamaica and the Philippines, and of different haplotypes in families originating from the same area (ie the two Italian families), also argues in favour of multiple origins. In Europe, three different haplotypes $A-4-6, B-3-6$ and $A-3-6$ possibly reflect three different founders.

\section{Haplotypes are Shared by Families from Common Geographic Areas}

Partial haplotypes were conserved among families with common geographic origins (North Africa, Korea, continental Europe and Anglo-Saxon countries). This could result from regional founder effects or from recurrent mutations on at-risk haplotypes, which differ according to geographic area. Regional founder effects appear to be common in spinocerebellar ataxias and were previously shown for SCA $1,{ }^{24} \mathrm{SCA} 2,{ }^{25,26} \mathrm{SCA} 3 /$ $\mathrm{MJD}^{27-32}$ and SCA6. ${ }^{33}$

The observation that haplotypes were less conserved among families from continental Europe and AngloSaxon countries than from North Africa might be explained by more ancient founder mutations which would be associated with more recombination events, microsatellite mutations or single-base pair changes. The observation that haplotypes were less conserved for telomeric markers that are more distant from the $S C A 7$ locus is consistent with recombination events following the ancestral mutation, in particular if there was a hot spot of recombination in this region. David et $a l^{12}$ demonstrated a marked discrepancy between physical and genetic distances in the $S C A 7$ region, suggesting that this might be the case. The Tunisian haplotype which is identical to that of other North African pedigrees, except for the addition of a single CA repeat at $D 3 S 1287$, is probably an example of a microsatellite mutation on a conserved haplotype. Similarly, the haplotype segregating in Korean and Filipino kindreds differed by a single CA repeat at D3S1287.

\section{Relation between IA and SCA7-linked Haplotypes}

The SCA7 expansion is much more unstable during transmission between generations than other SCA loci (mean increase of +12 CAG rather than +1 or +2$)$. ${ }^{1}$ This instability, which results in anticipation of about 20 years, should have led to rapid extinction of this disorder. This has not been the case, however, because of some instability of large normal alleles or intermediate alleles (IA), particularly during paternal transmissions, that leads to de novo expansion. ${ }^{20}$ In Huntington's disease, de novo mutations that also occur on intermediate-sized alleles, were associated with the most common haplotype linked to the disease. ${ }^{34,35}$ In the spinocerebellar ataxias, the frequencies of the mutations are closely related to those of IA in different ethnic groups. ${ }^{36}$ Furthermore, large normal alleles and disease-linked chromosomes in SCA3 and DRPLA share similar haplotypes. ${ }^{31,37}$

\section{Do IAs share haplotypes with the SCA7 mutations?}

In SCA7, intermediate alleles, observed in three different populations, even in individuals of common origin (ie the Italian families) do not have the same haplotype. Different pools of IA that can give rise to the disease therefore exist. IAs were observed in Tunisia with the same haplotype as the $S C A 7$ mutation in a family from the same region ${ }^{20}$ and also resemble those found in the majority of North African SCA7 kindreds. In this Tunisian pedigree, an IA segregated in one branch of the kindred, whereas expansions were transmitted in

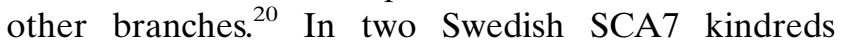
related to a single founder, ${ }^{14}$ the disease appeared independently, associated with similar haplotypes, five and eight generations later, probably by expansion of an IA transmitted by a common ancestor.

These data suggest that recurrent de novo expansions on at-risk haplotypes, derived from a founder chromosome which carries an IA, can account for the shared haplotypes observed in families from the same geographic area. De novo expansion on founder chromosomes has been demonstrated in the majority of the fragile $\mathrm{X}$ families of Finnish and Tunisian Jewish origins. ${ }^{38,39}$ More than one pool of at-risk chromosomes may be present in a single population, however, as illustrated by the two Italian families in the present study where IA giving rise to de novo mutations had different haplotypes.

\section{Acknowledgements}

The authors are grateful to the members of the families for their kind co-operation and to the clinicians who referred the patients: Drs S Belal, H Chneiweiss, S Cunha, D Grid, DB 
Hanna, AE Harding, W Horta, SG Jacobson, J Julien, JM Kwon, CH Lyoo, N Wood, M Yahyaoui and J Zlotogora. We would like to thank C Penet Y Pothin, A Camuzat and J Bou for technical assistance as well as $\mathrm{C}$ Zander and Drs $\mathrm{M}$ Ruberg and $\mathrm{J}$ Feingold for critical reading of the manuscript. The authors are indebted to Professor C Morocutti for allowing PG to examine patients from two Italian families. This work was funded by the Association RETINA/Rétinitis Pigmentosa, the Association Française contre les Myopathies, the VERUM Foundation, the Biomed concerted action (CEE) and the Association pour de Développement de la Recherche sur les Maladies Génétiques Neurologiques et Psychiatriques. GS is the recipient of a fellowship from the Fondation pour la Recherche Médicale. PG is supported by a grant from the European Neurological Society.

\section{References}

1 Stevanin G, David G, Abbas N et al: Spinocerebellar Ataxia 7. In: Rubinsztein DC, Hayden M (eds). Trinucleotide Repeat Diseases. Bios Scientific Publisher: Oxford, 1998, 155-168.

2 Harding AE: Clinical features and classification of inherited ataxias. Adv Neurol 1993; 61: 1-14.

3 Benomar A, Le Guern E, Dürr A et al: Autosomaldominant cerebellar ataxia with retinal degeneration (ADCA type II) is genetically different from ADCA type I. Ann Neurol 1994; 35: 439-444.

4 Enevoldson TP, Sanders MD, Harding AE: Autosomal dominant cerebellar ataxia with pigmentary macular dystrophy. A clinical and genetic study of eight families. Brain 1994; 117: 445-460.

5 Gouw LG, Digre KB, Harris CP, Haines JH, Ptacek LJ: Autosomal dominant cerebellar ataxia with retinal degeneration: clinical, neuropathologic, and genetic analysis of a large kindred. Neurology 1994; 44: 1441-1447.

6 David G, Abbas N, Stevanin G et al: Cloning of the SCA7 gene reveals a highly unstable CAG repeat expansion. Nat Genet 1997; 17: 65-70.

7 Del-Favero J, Krols L, Michalik A et al: Molecular genetic analysis of autosomal dominant cerebellar ataxia with retinal degeneration (ADCA type II) caused by CAG triplet repeat expansion. Hum Mol Genet 1998; 7: 177-186.

8 Koob MD, Benzow KA, Bird TDA, Day JW, Moseley ML, Ranum LPW: Rapid cloning of expanded trinucleotide repeat sequences from genomic DNA. Nat Genet 1998; 18: $72-75$.

9 Brice A: Unstable mutations and neurodegenerative disorders. J Neurol 1998; 245: 505-510.

10 Benton CS, de Silva R, Rutledge SL, Bohlega S, Ashizawa T, Zoghbi HY: Molecular and clinical studies in SCA-7 define a broad clinical spectrum and the infantile phenotype. Neurology 1998; 51: 1081-1086.

11 Dürr A, Cancel G, Stevanin G, David G, Didierjean O, Brice A: Features of autosomal dominant cerebellar ataxias with CAG repeat expansions. Am J Hum Genet 1998; 50 (suppl 4): S31.002.
12 David G, Giunti P, Abbas N et al: The gene for autosomal dominant cerebellar ataxia type II is located in a 5-cM region in 3p12-p13: genetic and physical mapping of the SCA7 locus. Am J Hum Genet 1996; 59: 1328-1336.

13 Gouw LG, Castaneda MA, McKenna CK et al: Analysis of the dynamic mutation in the $S C A 7$ gene shows marked parental effects on CAG repeat transmission. Hum Mol Genet 1998; 7: 525-532.

14 Johansson J, Forsgren L, Sandgren O, Brice A, Holmgren G, Holmberg M: Expanded CAG repeat in Swedish Spinocerebellar ataxia type 7 (SCA7) patients: effect of CAG repeat length on the clinical manifestation. Hum Mol Genet 1998; 7: 171-176.

15 Lyoo $\mathrm{CH}$, Hun K, Choi YC et al: CAG repeat expansion in the $S C A 7$ gene in Korean families presenting with ADCA type II. J Kor Neurol Ass 1998; 16: 341-352.

16 Giunti P, Stevanin G, Worth P, David G, Brice A, Wood NW: Molecular and clinical study of 18 families with ADCA type II: evidence for genetic heterogeneity and $d e$ novo mutation. Am J Hum Genet 1999; 64: 1594-1603.

17 Benomar A, Krols L, Stevanin G et al: The gene for autosomal dominant cerebellar ataxia with pigmentary macular dystrophy maps to chromosome 3p12-p21.1. Nat Genet 1995; 10: 84-88.

18 Jöbsis GJ, Weber JW, Barth PG et al: Autosomal dominant cerebellar ataxia with retinal degeneration (ADCA II): clinical and neuropathological findings in two pedigrees and genetic linkage to 3p12-p21.1. J Neurol Neurosurg Psychiatr 1997; 62: 367-371.

19 David G, Dürr A, Stevanin G et al: Molecular and clinical correlations in autosomal dominant cerebellar ataxia with progressive macular dystrophy (SCA7). Hum Mol Genet 1998; 7: 165-170.

20 Stevanin G, Giunti P, David G et al: De novo expansion of intermediate alleles in spinocerebellar ataxia 7. Hum Mol Genet 1998; 7: 1809-1813.

21 Krols L, Martin JJ, David G et al: Refinement of the locus for autosomal dominant cerebellar ataxia type II to chromosome 3p21.1-14.1. Hum Genet 1997; 99: 225-232.

22 Imbert G, Saudou F, Yvert G et al: Cloning of the gene for spinocerebellar ataxia 2 reveals a locus with high sensitivity to expanded CAG/glutamine repeats. Nat Genet 1996; 14: 285-291.

23 Feingold J: Le déséquilibre de liaison. Médecine/Sciences 1991; 7: 161-168.

24 Wakisaka A, Sasaki H, Takada A et al: Spinocerebellar ataxia 1 (SCA1) in the Japanese in Hokkaido may derive from a single common ancestry. J Med Genet 1995; 32: 590-592.

25 Mizushima K, Watanabe M, Kondo I et al: Analysis of spinocerebellar ataxia type 2 gene and haplotype analysis: (CCG)1-2 polymorphism and contribution to founder effect. J Med Genet 1999; 36: 112-114.

26 Didierjean O, Cancel G, Stevanin G et al: Linkage disequilibrium at the SCA2 locus. J Med Genet 1999; 36: 415-417.

27 Stevanin G, Cancel G, Didierjean O et al: Linkage disequilibrium at the Machado-Joseph disease/Spinal cerebellar ataxia 3 locus: evidence for a common founder effect in French and Portuguese-Brazilian families as well as a second ancestral Portuguese-Azorean mutation. Am J Hum Genet 1995; 57: 1247-1250. 
28 Takiyama Y, Igarashi S, Rogaeva EA et al: Evidence for inter-generational instability in the CAG repeat in the MJD1 gene and for conserved haplotypes at flanking markers amongst Japanese and Caucasian subjects with Machado-Joseph disease. Hum Mol Genet 1995; 4: 1137-1146.

29 Gaspar C, Lopes-Cendes I, DeStefano AL et al: Linkage disequilibrium analysis in Machado-Joseph disease patients of different ethnic origins. Hum Genet 1996; 98: 620-624.

30 Endo K, Sakashi H, Wakisaka A et al: Strong linkage disequilibrium and haplotype analysis in Japanese pedigrees with Machado-Joseph disease. Am J Med Genet 1996; 67: 437-444.

31 Stevanin G, Lebre AS, Mathieux C et al: Linkage disequilibrium between the spinocerebellar ataxia 3/ Machado-Joseph disease mutation and two intragenic polymorphisms, one of which, X359Y, affects the stop codon. Am J Hum Genet 1997; 60: 1548-1552.

32 Goto J, Watanabe M, Ichikawa Y et al: Machado-Joseph disease gene products carrying different carboxyl termini. Neurosci Res 1997; 28: 373-377.

33 Dichgans M, Schols L, Herzog J et al: Spinocerebellar ataxia type 6: evidence for a strong founder effect among German families. Neurology 1999; 52: 849-851.

34 Myers RH, MacDonald ME, Koroshetz WJ et al: De novo expansion of a (CAG)n repeat in sporadic Huntington's disease. Nat Genet 1993; 5: 168-173.
35 Almqvist E, Spence N, Nichol K et al: Ancestral differences in the distribution of the delta 2642 glutamic acid polymorphism is associated with varying CAG repeat lengths on normal chromosomes: insights into the genetic evolution of Huntington disease. Hum Mol Genet 1995; 4: 207-214.

36 Takano H, Cancel G, Ikeuchi T et al: Population genetics of dominantly inherited spinocerebellar ataxias with CAG repeat expansions in Japanese and Caucasian: tight association of the prevalence rates with the frequencies of intermediate alleles. Am J Hum Genet 1998; 63: 1060-1066.

37 Yanagisawa H, Fujii K, Nagafuchi S et al: A unique origin and multistep process for the generation of expanded DRPLA triplet repeats. Hum Mol Genet 1996; 5: 373-379.

38 Oudet C, Mornet E, Serre JL et al: Linkage disequilibrium between the fragile $\mathrm{X}$ mutation and two closely linked CA repeats suggests that fragile $\mathrm{X}$ chromosomes are derived from a small number of founder chromosomes. Am J Hum Genet 1993; 52: 297-304.

39 Falik-Zaccai TC, Shachak E, Yalon M et al: Predisposition to the Fragile $\mathrm{X}$ syndrome in Jews of Tunisian descent is due to the absence of AGG interruptions on a rare Mediterranean haplotype. Am J Hum Genet 1997; 60: 103-112. 